\title{
SUSTAINING TURBULENCE IN SPECTRALLY STABLE SHEAR FLOWS - INTERPLAY OF LINEAR TRANSIENT GROWTH AND NONLINEAR TRANSVERSE CASCADE
}

\author{
D. Gogichaishvili ${ }^{1}$, G. Mamatsashvili ${ }^{2}$, W. Horton ${ }^{3}$ and G. Chagelishvili ${ }^{4}$
}

\begin{abstract}
We analyze the sustaining mechanism of nonlinear perturbations/turbulence in spectrally stable smooth shear flows. The essence of the sustenance is a subtle interplay of linear transient growth of Fourier harmonics and nonlinear processes. In spectrally stable shear flows, the transient growth of perturbations is strongly anisotropic in spectral (k-)space. This, in turn, leads to anisotropy of nonlinear processes in $\mathbf{k}$-space and, as a result, the main (new) nonlinear process appears to be not a direct/inverse, but rather a transverse/angular redistribution of harmonics in Fourier space referred to as the nonlinear transverse cascade. It is demonstrated that nonlinear state is sustained owing to the interplay of the linear nonmodal growth and the transverse cascade. The possibility of such course of events has been described in k-space by G. Chagelishvili, J.-P. Zahn, A. Tevzadze and J. Lominadze, A\&A, 402, 401 (2003) that reliably exemplifies the well-known bypass scenario of subcritical turbulence in spectrally stable shear flows. We present selected results of the simulations performed in different (HD and MHD; 2D and 3D; plane and Keplerian) shear flows to demonstrate the transverse cascade in action.
\end{abstract}

\section{Introduction}

Developments in understanding the physics of the causes of turbulence is astrophysical disks have been irregular and taken considerable time. Delay in the comprehension of turbulence in astrophysical disks was due to some inadequacy of the canonical/modal/spectral approach to study linear stability of smooth shear

\footnotetext{
${ }^{1}$ Department of Physics, The University of Texas at Austin, Austin, Texas 78712, USA

2 Helmholtz-Zentrum Dresden-Rossendorf, P.O. Box 510119, 01314 Dresden, Germany

${ }^{3}$ Institute for Fusion Studies, The University of Texas at Austin, Austin, Texas 78712, USA

4 Abastumani Astrophysical Observatory, Ilia State University, Tbilisi 0162, Georgia
} 
flows, which ultimately led to the change of paradigm to a more general nonmodal approach (Schmid \& Henningson 2000). Now the phrases: shear flow nonnormality, nonmodal approach, transient growth, bypass concept of turbulence, shearing sheet and etc. are already a common parlance in the fluid dynamical and astrophysical disk communities. Although, retrospectively a thinking inertia pretty long was clutching on the modal approach - eigenfunctions and eigenvalues - and delayed the legitimation of these concepts in studies of dynamical processes in disks.

In the beginning of the 1990s, the non-normal nature of shear flows and its consequences became well understood and extensively studied by the hydrodynamic community (Reddy \& Henningson 1993; Trefethen et al. 1993; Schmid \& Henningson 2000). Shortcomings of traditional modal analysis (spectral expansion of perturbations in time and subsequent analysis of eigenfunctions) for shear flows have been revealed. Operators in the mathematical formalism of the modal analysis are non-normal and the corresponding eigenmodes are nonorthogonal (Reddy \& Henningson 1993; Trefethen \& Embree 2005; Schmid 2007). The nonorthogonality leads to strong interference among the eigenmodes. Consequently, a proper approach should fully analyze this interference. While being in principle possible, this is in practice a formidable task. On this issue colorfully expressed L. Trefethen (1991): "For non-normal systems eigenvalues may still be used to a greater degree, like a nail file when a screwdriver can't be found, but they are rarely exactly right". As a result, the mathematical approach was shifted from the 1990s to the nonmodal approach and a breakthrough in the understanding and precise description of linear transient phenomena ensued. In spectrally stable hydrodynamic shear flows, it became clear that vortex and wave mode perturbations of certain spatial characteristics/orientation undergo linear transient growth (Gustavsson 1991; Farrell \& Ioannou 1993; Chagelishvili et al. 2003), leading to short perturbation life times. However, in certain favorable circumstances, the perturbations are sustained for long time by the shear flow. Specifically, the imperfect linear transient growth, which is the only source of energy for turbulence in such flows, must be compensated by the nonlinear positive feedback, i.e. the role of nonlinear processes becomes crucial: they lie at the heart of sustenance of the turbulence - close the feedback loop ensuring the sustaining dynamics. On the basis of this interplay between linear transient growth and nonlinear positive feedback, the hydrodynamic community formulated the concept of bypass transition to explain the onset and sustenance of turbulence in spectrally stable shear flows (see, e.g. Baggett et al. 1995; Grossmann 2000; Chapman 2002). However, the bypass concept is usually illustrated on toy models (Trefethen et al. 1993) or on phenomenological analysis in physical space (Waleffe 1995). So, it seems appropriate to consider the simplest/constant shear flow and analyze the interplay of the linear transient and nonlinear feedback processes in spectral (k-)space. First, a constant shear flow is not an idealization, but quite a realistic/useful model. For instance, the well-known shearing sheet approximation for investigation of local dynamics in astrophysical disks is based on the background flow configuration with constant shear of velocity. Second, canonical nonlinear processes, such as 
direct/inverse cascade, are analyzed in spectral space. Therefore, it is natural to investigate how enriched the canonical nonlinear processes are by flow shear, whether the direct and inverse cascades are modified, or a new type of cascade process appears. The following thoughts can contribute to the clarification of these questions. Actually, the shear-induced linear nonmodal growth of a perturbation harmonic mainly depends on its wave vector orientation and, to a lesser degree, on the value (e.g. Farrell 1988; Butler \& Farrell 1992; Farrell \& Ioannou 1993; Jiménez 2013): the spatial Fourier harmonics that have a certain orientation of the wave vector with respect to shear flow draw energy from it and grow, whereas harmonics with other orientation of the wave vector give energy to the flow and decay. This anisotropy of the linear energy-exchange processes with respect to wave vector orientation (angle), in turn, leads to anisotropy of nonlinear processes in k-space. Specifically, as revealed in Horton et al. (2010), Mamatsashvili et al. (2014, 2016), in HD and MHD smooth shear flows, the main nonlinear process is not a direct or inverse, but rather, so-called, nonlinear transverse cascade, that is angular (transverse) redistribution of perturbation harmonics in $\mathbf{k}$-space. The nonlinear transverse cascade, or transverse cascade for short, represents an alternative to the canonical direct and inverse cascades in the presence of flow velocity shear. Such angular redistribution of harmonics in $\mathbf{k}$-space has been elaborated in collaboration with J.-P. Zahn in the context of the problem of hydrodynamic shear turbulence in non-magnetized Keplerian disks (Chagelishvili et al. 2003). This study first showed the applicability/relevance of the findings of the hydrodynamic community for spectrally stable shear flows, the nonorthogonality of linear operators and the nonmodal approach, the mechanism of perturbation amplification and the bypass concept of onset of turbulence for the dynamics of disks. Then, a simple sketch of the bypass scenario in wavenumber $\left(k_{x}, k_{y}\right)$-plane was presented (see Fig. A.1 of Chagelishvili et al. 2003), which schematically describes the nonlinear angular redistribution of harmonics, i.e. the transverse cascade. Other two sequel papers of J.-P. Zahn of this series Tevzadze et al. (2003, 2008) are also impregnated with the spirit of the transverse cascade. Subsequently, a key character of the transverse cascade was confirmed and analyzed for different $2 \mathrm{D}$ and $3 \mathrm{D}$ constant shear flows using numerical simulations (Horton et al. 2010; Mamatsashvili et al. 2014, 2016). Nevertheless, apparently, the thinking inertia still persists with respect to this new type of nonlinear cascade. Indeed, there are quite a few papers, where, studying astrophysical disk turbulence in the local shearing sheet setup, so-called, "shell-averaging" is used in k-space, which consists in averaging spectral quantities/processes over azimuthal and meridional angles of wave vector and representing as functions of only its magnitude $|\mathbf{k}|$, thereby whipping out the spectral anisotropy of the dynamics. Of course, this eliminates the transverse cascade - a key factor of turbulence's sustenance in the disks. In this respect, in this limited length paper, we intend to show as vividly as possible the nonlinear transverse cascade in action. In Section 2, we describe the dynamics of coherent cyclonic and anticyclonic vortices in 2D HD plane constant shear flow. The coherence of perturbations leads to a regular, easily recognizable nonlinear transverse/ angular redistribution of harmonics in $\left(k_{x}, k_{y}\right)$-plane, i.e. the transverse cascade. 
At the same time, we present the corresponding mathematical scheme, clarifying the interplay of the linear transient and nonlinear transverse cascade processes. In Sections 3 and 4, sustained MHD homogeneous turbulence in spectrally stable 2D (plane) and 3D (Keplerian) shear flows with uniform magnetic field parallel to the flow is studied in $\mathbf{k}$-space.

\section{Coherent vortices in 2D plane shear flows - transverse cascade}

The nonlinear transverse cascade and its interplay with the linear transient growth can be clearly demonstrated in $\left(k_{x}, k_{y}\right)$-plane by analyzing the dynamics of coherent cyclonic and anticyclonic vortices in 2D homogeneous constant shear flow, $U_{0}(x)=(0, A x)$, with the shear parameter $A>0$ (Horton et al. 2010). The nonlinear dynamical equation for the stream function of perturbations, $\psi$, is

$$
\left[\frac{\partial}{\partial t}+U_{0}(x) \frac{\partial}{\partial y}\right] \Delta \psi+J(\psi, \Delta \psi)-\nu \Delta^{2} \psi=0,
$$

where the spatial operators $J(.,$.$) and \Delta$ are, respectively, 2D Jacobian and Laplacian and $\nu$ is the viscosity. The velocity and energy density of perturbations are defined through $\psi$ and density $\rho$, respectively, as

$$
v_{x}=-\frac{\partial \psi}{\partial y} ; \quad v_{y}=\frac{\partial \psi}{\partial x}, \quad e(x, y, t)=\frac{1}{2} \rho\left[\left(\frac{\partial \psi}{\partial y}\right)^{2}+\left(\frac{\partial \psi}{\partial x}\right)^{2}\right] .
$$

Initially we impose coherent vortices with Gaussian shape of stream function in the $(x, y)$-plane:

$$
\left.\psi(x, y, t)\right|_{t=0}=n b \exp \left(-\frac{x^{2}+y^{2}}{l^{2}}\right) .
$$

where $n=-1$ corresponds to cyclonic and $n=1$ to anticyclonic vortex perturbations and $b>0$ is their amplitude. Introduce non-dimensional parameters,

$$
\tau \equiv A t, \quad(X, Y) \equiv \frac{(x, y)}{l}, \quad B \equiv \frac{b}{A l^{2}}, \Psi \equiv \frac{\psi}{A l^{2}}, \quad E \equiv \frac{2 e}{\rho A^{2} l^{2}}, \quad R e \equiv \frac{A l^{2}}{\nu},
$$

and perform Fourier transform with respect to the coordinates $X$ and $Y$,

$$
\Psi=\int d k_{x} d k_{y} \Psi_{\mathbf{k}} \exp \left(i k_{x} X+i k_{y} Y\right) .
$$

Substituting Equation (2.3) into Equations (2.1) and (2.2), one can obtain the evolution equation for $\Psi_{\mathbf{k}}$ :

$$
k^{2} \frac{\partial \Psi_{\mathbf{k}}}{\partial \tau}-k_{y} \frac{\partial\left(k^{2} \Psi_{\mathbf{k}}\right)}{\partial k_{x}}+\frac{k^{4}}{R e} \Psi_{\mathbf{k}}=\sum_{\mathbf{k}=\mathbf{k}^{\prime}+\mathbf{k}^{\prime \prime}}\left(k_{x}^{\prime} k_{y}^{\prime \prime}-k_{x}^{\prime \prime} k_{y}^{\prime}\right) k^{\prime 2} \Psi_{\mathbf{k}^{\prime}} \Psi_{\mathbf{k}^{\prime \prime}},
$$

with the corresponding initial condition in Fourier plane

$$
\left.\Psi_{\mathbf{k}}\right|_{\tau=0}=n B \exp \left(-\frac{k_{x}^{2}+k_{y}^{2}}{4 \pi^{2}}\right)
$$




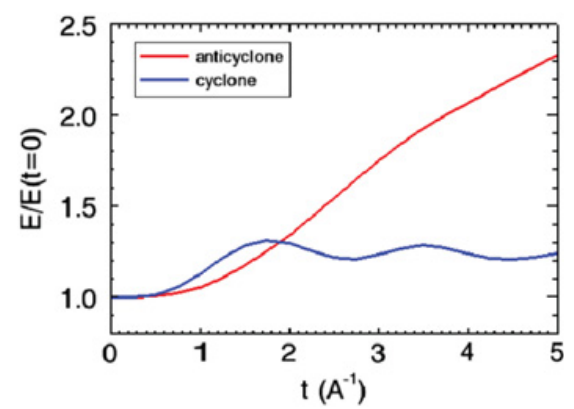

Fig. 1. Normalized total energy vs. time for coherent anticyclonic (red) and cyclonic (blue) vortex perturbations for $R e=1000$ and $B=3$. The energy of the anticyclonic vortex increases monotonically, while the energy of the cyclonic one initially increases and then oscillates weakly around a constant value.

where $k^{2} \equiv k_{x}^{2}+k_{y}^{2}$. The nonlinear term on the right hand side (rhs) of this equation describes three-wave interactions. Equation (2.4) forms the basis of the numerical study to explore quantitatively the dynamics of the stream function, spectral energy density, and total energy of cyclonic and anticyclonic vortices. However, to investigate the physics of the phenomena, one has to analyze the dynamical equation for the spectral kinetic energy density, $E_{\mathbf{k}}=k^{2}\left|\Psi_{\mathbf{k}}\right|^{2}$, which follows from Equation (2.4),

$$
\frac{\partial E_{\mathbf{k}}}{\partial \tau}=k_{y} \frac{\partial E_{\mathbf{k}}}{\partial k_{x}}+\frac{2 k_{x} k_{y}}{k^{2}} E_{\mathbf{k}}-\frac{k^{2}}{R e} E_{\mathbf{k}}+N_{\mathbf{k}},
$$

where $N_{\mathbf{k}}$ is the nonlinear term:

$$
N_{\mathbf{k}}=\sum_{\mathbf{k}=\mathbf{k}^{\prime}+\mathbf{k}^{\prime \prime}}\left(k_{x}^{\prime} k_{y}^{\prime \prime}-k_{x}^{\prime \prime} k_{y}^{\prime}\right) k^{2}\left(\Psi_{\mathbf{k}}^{*} \Psi_{\mathbf{k}^{\prime}} \Psi_{\mathbf{k}^{\prime \prime}}+\Psi_{\mathbf{k}} \Psi_{\mathbf{k}^{\prime}}^{*} \Psi_{\mathbf{k}^{\prime \prime}}^{*}\right)
$$

The terms on the rhs of Equation (2.5) represent four - three linear and one nonlinear - basic phenomena: 1 . The linear flux of the spectral kinetic energy parallel to the $k_{x}$-axis, 2 . The energy exchange between the perturbation harmonics and the background flow, 3. The viscous dissipation, 4. The nonlinear processes that redistribute the energy of perturbation harmonics in $\mathbf{k}$-plane, but do not change their total energy. The interplay of these basic phenomena defines $\partial E_{\mathbf{k}} / \partial \tau$. Solving numerically Equation (2.4), or equivalently Equation (2.5), one can get full information on all the physical quantities and the basic phenomena.

The evolution of the perturbation energy normalized to the initial value $E / E_{0}$ for the coherent anticyclonic and cyclonic perturbations are presented in Figure 1. The energy of the anticyclonic vortex increases monotonically, while the energy of the cyclonic one initially increases and then oscillates weakly around a constant value. Since, the linear dynamics of the perturbations are identical, the difference in the energy evolutions is due to the nonlinear phenomena described in $\left(k_{x}, k_{y}\right)$ plane by $N_{\mathbf{k}}$. This term for the anticyclonic vortex (with $B=3$ and $R e=1000$ ) 


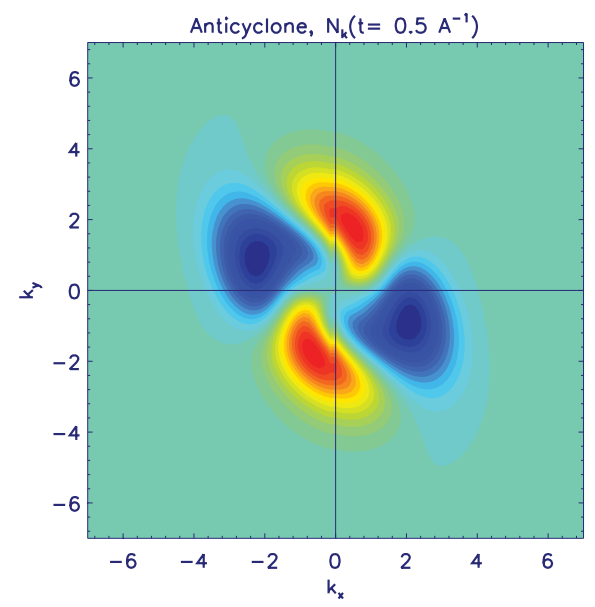

(a)

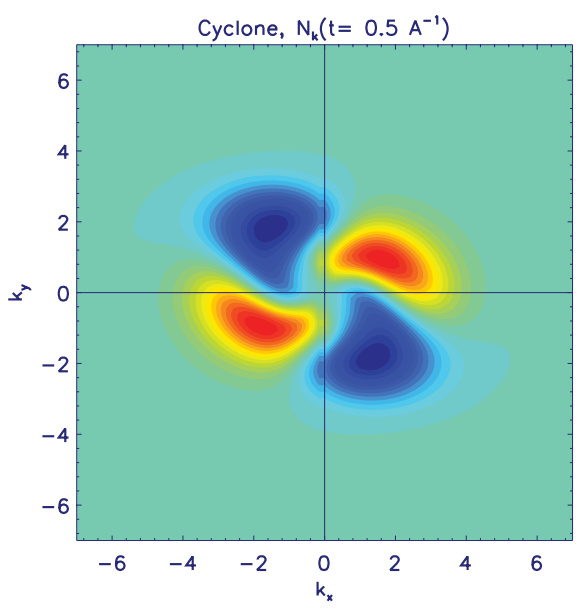

(a)

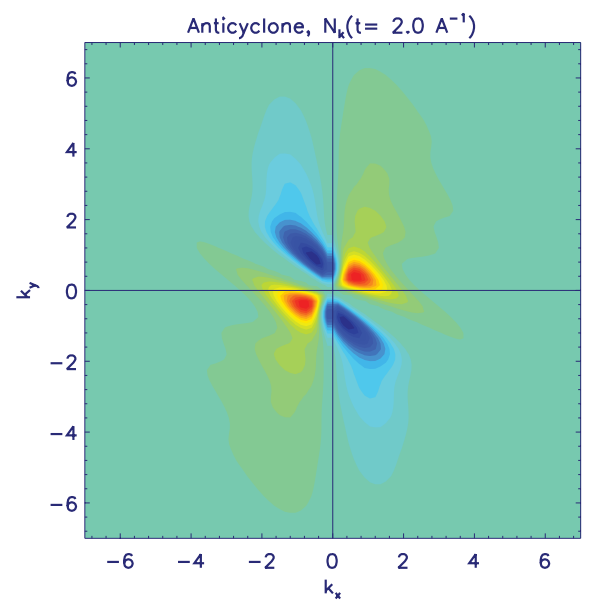

(b)

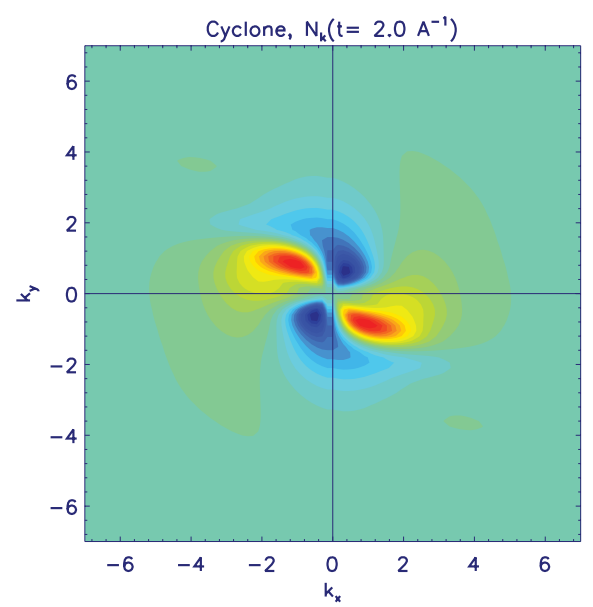

(b)

Fig. 2. The spectrum of the nonlinear term $N_{\mathbf{k}}\left(k_{x}, k_{y}, \tau\right)$ for the coherent anticyclonic (upper two plots) and cyclonic (lower two plots) vorticies with $R e=1000$ and $B=3$ at times $\tau=0.5$ and 2 , respectively. The nonlinearity redistributes the perturbation harmonics energy from the blue areas (where $N_{\mathbf{k}}<0$ ) to the red ones (where $N_{\mathbf{k}}>0$ ) the nonlinear transverse cascade is strongly pronounced.

is presented in upper plots of Figure 2 at $\tau=0.5$ and 2 . The nonlinearity redistributes the perturbation energy from the blue areas (where $N_{\mathbf{k}}<0$ ) to the red ones (where $N_{\mathbf{k}}>0$ ). The green refers to areas where $N_{\mathbf{k}} \simeq 0$. As one can see, the nonlinear interactions redistribute energy from quadrants $I I$ and $I V$ (where $N_{\mathbf{k}}$ is predominantly negative), to quadrants $I$ and $I I I$ (where $N_{\mathbf{k}}$ is predominantly positive). This nonlinear angular redistribution, or transverse cascade, 
repopulates the growing harmonics, which extract shear flow energy and are amplified in quadrants $I$ and $I I I$ of k-plane, where $k_{x} k_{y}>0$ (see Eq. (2.5)). This nonlinear feedback mechanism leads to the growth of the total energy of the coherent anticyclonic perturbations (the red curve in Fig. 1), i.e. - to the sustenance of anticyclonic perturbations. Also, one can see that the domain of significant nonlinear activity shrinks in time towards lower wave numbers: an inverse cascade is also at work. However, the dominant nonlinear process is the transverse cascade.

The lower plots of Figure 2 show that the transverse cascade is also strongly pronounced for coherent cyclonic vortices. However, in this case the nonlinear dynamics is more complicated. Positive and negative feedbacks alternate in time. Indeed, we see that nonlinear interactions redistribute energy mainly over wavenumber angle, from quadrants $I I$ and $I V$ to quadrants $I$ and $I I I$ at $\tau=0.5$ and vice versa at $\tau=2$. Hence, the transverse cascade repopulates growing harmonics (for which $k_{x} k_{y}>0$ ) at $\tau=0.5$, contributing to an increase in the cyclonic vortex total energy. By contrast, the repopulation of decaying harmonics by the transverse cascade leads to a decrease in the total energy at $\tau=2$ (see variation of the blue curve in Fig. 1). So, in the case of the cyclonic vortex, the transverse cascade does not ensure a continuous positive feedback.

\section{Sustaining turbulence in spectrally stable 2D MHD plane shear flows}

In this section, following Mamatsashvili et al. (2014), we demonstrate manifestations of the transverse cascade for sustained 2D MHD turbulence in incompressible constant shear flow, $\mathbf{U}_{0}=(0,-A x)$, threaded by an uniform background magnetic field parallel to the flow, $\mathbf{B}_{0}=\left(0, B_{0 y}\right)$ (without loss of generality, $\left.A, B_{0 y}>0\right)$. This flow is spectrally stable and hence the turbulence is subcritical by definition, being supported energetically only by a transient growth mechanism due to shear flow non-normality. As for the essence of the nonlinear processes, it is again a transverse redistribution of kinetic and magnetic spectral energies. We present the results of direct numerical simulations in $\left(k_{x}, k_{y}\right)$-plane, demonstrating the dominance of the transverse cascade in 2D MHD plane shear flows too.

We consider basic equations of non-ideal incompressible MHD flow and use normalized variables by taking the shear time, $A^{-1}$, as the unit of time; the Alfvén speed, $u_{A}=B_{0 y} /(4 \pi \rho)^{1 / 2}$, as the unit of velocity; $\ell \equiv u_{A} A^{-1}$ as the unit of length; and $B_{0 y}$, as the unit of the magnetic field perturbations (see details in Mamatsashvili et al. 2014). Figure 3 shows the time-development of the domainaveraged perturbed kinetic, $\left\langle E_{K}\right\rangle$, and magnetic, $\left\langle E_{M}\right\rangle$, energies as well as the Reynolds, $\left\langle u_{x} u_{y}\right\rangle$, and Maxwell $-\left\langle b_{x} b_{y}\right\rangle$ stresses. After growth, the energies and stresses settle down to a quasi-steady state of sustained turbulence. In this state, the kinetic and magnetic energies are comparable. The Maxwell stress is much larger than the Reynolds stress, indicating that the turbulent transport and energy extraction from the mean flow are ensured by the magnetic field perturbations the Maxwell stress counteracts dissipative processes and plays a decisive role in the maintenance of the turbulence. Therefore, we analyze only dynamical equation for 

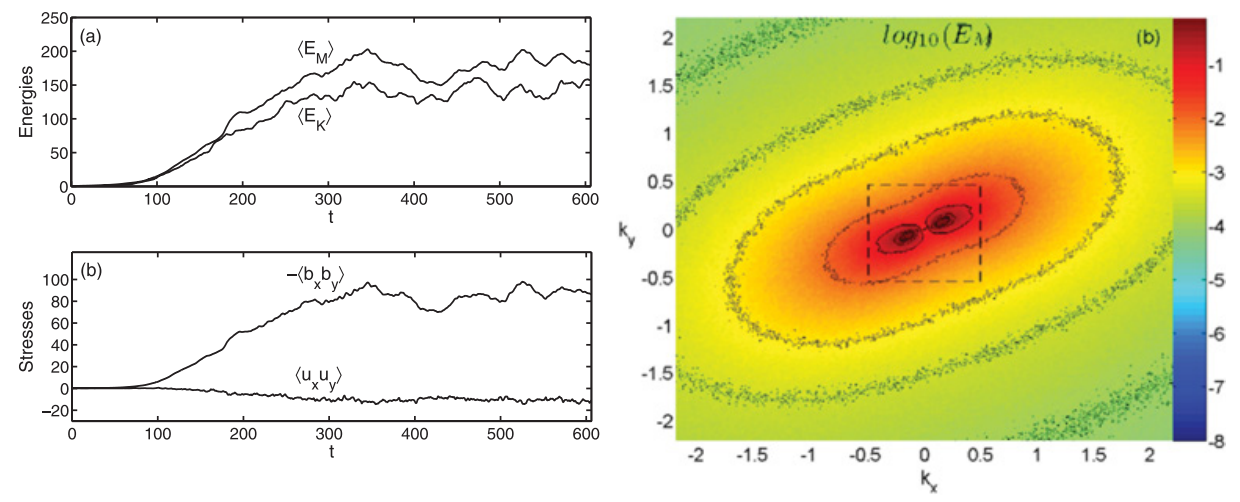

Fig. 3. Left: the domain-averaged (a) perturbed kinetic, $\left\langle E_{K}\right\rangle$, and magnetic, $\left\langle E_{M}\right\rangle$, energies as well as (b) the Reynolds and Maxwell stresses vs. time. Right plot: Logarithm of the time-averaged magnetic energy spectra in $\left(k_{x}, k_{y}\right)$-plane in the quasi-stationary turbulent state. The dashed rectangle encloses the central, small wavenumber area of $\left(k_{x}, k_{y}\right)$-plane that is vital for the sustenance of the turbulence.

nondimensional magnetic spectral energy, $E_{M}=\left|b_{x}\right|^{2}+\left|b_{y}\right|^{2}$ :

$$
\frac{\partial E_{M}}{\partial t}+k_{y} \frac{\partial E_{M}}{\partial k_{x}}=I_{M}+I_{M K}+D_{M}+N_{M},
$$

where, the second term on the left hand side is the flux of the spectral magnetic energy parallel to $k_{x}$ axis, $I_{M}$ is the Maxwell stress spectrum, responsible for the magnetic energy exchange with the mean flow and perturbation harmonics, $I_{M K}$ is the exchange between the kinetic and magnetic spectral energies, $D_{M}$ is the dissipation of magnetic spectral energy due to resistivity and $N_{M}$ is the nonlinear transfer of the magnetic spectral energy in $\left(k_{x}, k_{y}\right)$-plane:

$$
\begin{gathered}
I_{M}=-b_{x} b_{y}^{*}-\text { c.c. }=\frac{2 k_{x} k_{y}}{k^{2}} E_{M}, I_{M K}=\mathrm{i} k_{y}\left(u_{x} b_{x}^{*}+u_{y} b_{y}^{*}-\text { c.c. }\right), D_{M}=-\frac{2 k^{2}}{R m} E_{M} \\
N_{M}(\mathbf{k}, t)=\mathrm{i}\left(k_{y} b_{x}^{*}-k_{x} b_{y}^{*}\right) \sum_{\mathbf{k}=\mathbf{k}^{\prime}+\mathbf{k}^{\prime \prime}}\left[u_{x}\left(\mathbf{k}^{\prime}, t\right) b_{y}\left(\mathbf{k}^{\prime \prime}, t\right)-u_{y}\left(\mathbf{k}^{\prime}, t\right) b_{x}\left(\mathbf{k}^{\prime \prime}, t\right)\right]+\text { c.c. }
\end{gathered}
$$

The simulations have shown that $I_{M K} \ll I_{M}$, therefore, in Figures 3 and 4 we present only plots of time-averaged $E_{M}, I_{M}$ and $N_{M}$ in $\left(k_{x}, k_{y}\right)$-plane in the quasi-steady turbulent state to understand the character of the above anisotropic magnetic energy spectra and nonlinear transfers. Like the spectral energy (Fig. 3), $I_{M}$ and $N_{M}$ (Fig. 4) also exhibit anisotropy over wavenumbers - they are inclined towards the $k_{x}$-axis due to shear. $I_{M}$ mostly operates at wavenumbers $0.05<$ $k<0.5$ and is dominant and positive on the $k_{x} / k_{y}>0$ side (red and yellow regions), where it supplies harmonics with magnetic energy. The net result of $I_{M}$ 

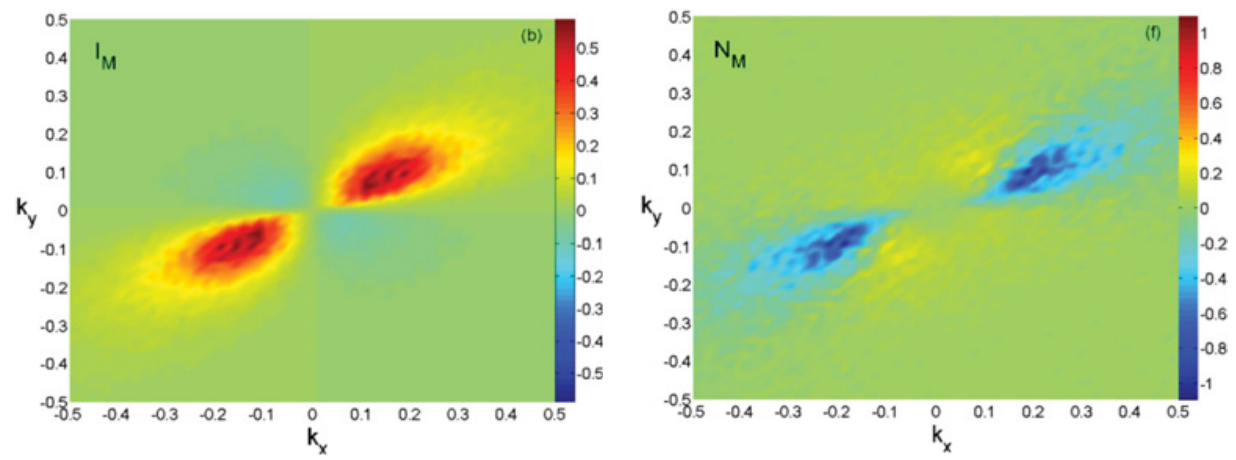

Fig. 4. The time-averaged spectra of $I_{M}$ (left) and the nonlinear transfer term $N_{M}$ (right) in $\mathbf{k}$-plane in the state of quasi-steady turbulence. $N_{M}$ transfers magnetic energy anisotropically (transversely) in wavenumber plane, away from regions where $N_{M}<0$ (blue) to regions where $N_{M}>0$ (yellow).

over all wavenumbers is a positive energy gain for perturbations (i.e. $\left.\left\langle-b_{x} b_{y}\right\rangle>0\right)$ that maintains the turbulence. Consider harmonics located left to the injection region, where $I_{M}>0$. These harmonics drift along the $k_{x}$-direction and enter the injection region. As a result, their magnetic energy grows at $k_{y}>0.05$ at the expense of the mean flow. Then, the harmonics move into the regions where $N_{M}<0$ (blue) and hence these nonlinear terms now act to transfer part of the magnetic energy gained back, respectively, to the regions where $N_{M}>0$ (yellow), from which these harmonics started off, in this way regenerating new harmonics there (positive nonlinear feedback). During each cycle, the harmonics gain the magnetic energy from the mean flow due to the injection term $I_{M}$. Part of this magnetic energy is transformed by nonlinearity into the kinetic and the other part into magnetic energies of the newly created harmonic; the rest is dissipated due to resistivity. The new (regenerated) harmonics will drift through the same cycle and the whole process of (magnetic) energy extraction from the mean flow will be repeated. In this way, a positive feedback loop - a cooperative interplay of the linear transient amplification and nonlinear transverse redistribution of the magnetic spectral energy - is established, ensuring the sustenance of a quasi-steady turbulent state at the expense of the background shear flow energy.

\section{Sustaining MHD turbulence in spectrally stable Keplerian disk flows}

In this final section, we present the nonlinear transverse cascade in action in the disk flow with: (i) Keplerian rotation in the shearing sheet approximation, $\mathbf{U}_{0}=$ $(0,-q \Omega x, 0)$, where $\Omega$ is the local angular velocity of rotation and $q=1.5$ is the normalized Keplerian shear parameter, (ii) azimuthal uniform magnetic field, $\mathbf{B}_{0}=\left(0, B_{0 y}, 0\right)$, and (iii) vertical stratification. Due to the limited space, we characterize mostly the nonlinear transverse cascade, albeit in highly compressed 

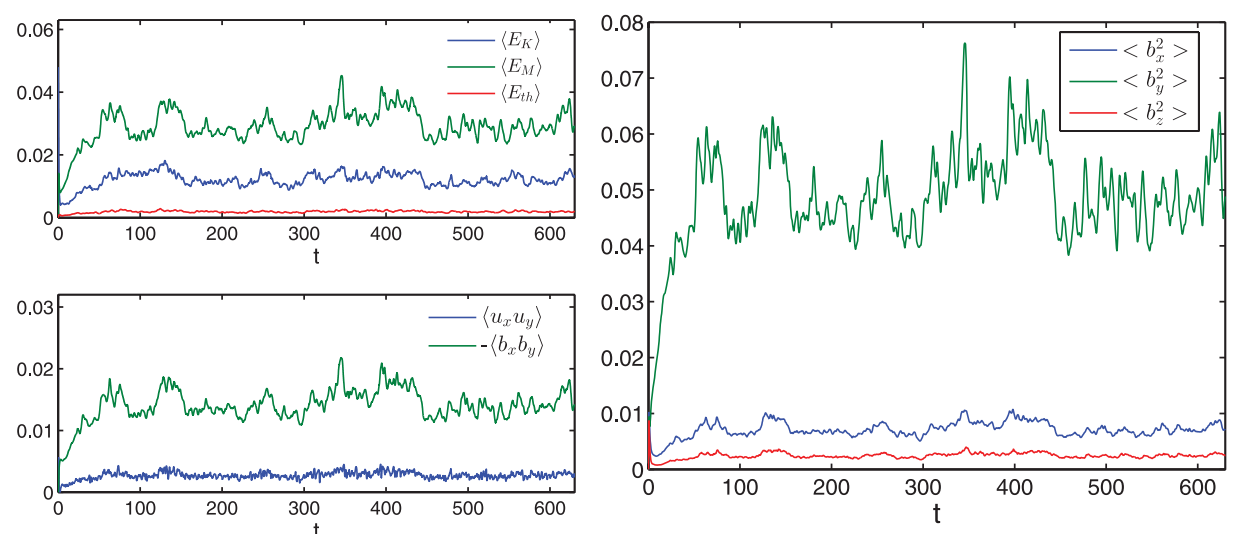

Fig. 5. Left: Evolution of the volume-averaged kinetic, $\left\langle E_{K}\right\rangle$, magnetic, $\left\langle E_{M}\right\rangle$, and thermal, $\left\langle E_{t h}\right\rangle$, energies as well as the Reynolds, $\left\langle u_{x} u_{y}\right\rangle$, and Maxwell, $\left\langle-b_{x} b_{y}\right\rangle$, stresses. Right: Evolution of the volume-averaged turbulent field components, with $\left\langle b_{y}^{2}\right\rangle$ dominating the other two.

form. It is justified by the fact that, despite the more complex flow system, the transverse cascade and its interplay with the transient (nonmodal) growth process ensures the sustenance of turbulence and proceeds very similarly to that in the above cases. This fact indicates the universal and key character of this (new type of) cascade in shear flows.

Figure 5 shows that in quasi-stationary turbulent state, the magnetic energy and Maxwell stress exceed several times the kinetic energy and Reynolds stress, respectively. The thermal energy is even much less. Therefore, we restrict the analysis of the dynamics to the quadratic forms of magnetic field perturbations in k-space, $\left|b_{x}\right|^{2},\left|b_{y}\right|^{2}$ and $\left|b_{z}\right|^{2}$ ), which are governed by the following dynamical equations:

$$
\frac{\partial}{\partial t}\left|b_{i}\right|^{2} / 2+q k_{y} \frac{\partial}{\partial k_{x}}\left|b_{i}\right|^{2} / 2=q \mathcal{M} \delta_{i y}+\mathcal{I}_{i}^{(u b)}+\mathcal{D}_{i}^{(b)}+\mathcal{N}_{i}^{(b)}, \quad i=x, y, z
$$

where $\mathcal{M}=-\left(b_{x} b_{y}^{*}+b_{x}^{*} b_{y}\right) / 2$ is the Maxwell stress spectrum; $\mathcal{I}_{i}^{(u b)}=\mathrm{i} k_{y} B_{0 y} u_{i} b_{i}^{*} /$ $2+$ c.c. is the exchange term between velocity $\left(u_{i}\right)$ and magnetic field $\left(b_{i}\right) ; \mathcal{D}_{i}^{(b)}$ and $\mathcal{N}_{i}^{(b)}$ are $i$ components of dissipative and nonlinear terms, respectively. The second term on the left hand side leads to drift of $\left|b_{i}\right|^{2}$ in $\mathbf{k}$-space parallel to the $k_{x}$-axis right, if $k_{y}>0$ and left, if $k_{y}<0$. These equations show that the Maxwell stress energetically supports only $\left|b_{y}\right|^{2}$. $\left|b_{x}\right|^{2}$ and $\left|b_{z}\right|^{2}$ should be supported by $\mathcal{I}_{i}^{(u b)}$ and $\mathcal{N}_{i}^{(b)}$. Following analogous local (shearing sheet) studies of disk dynamics (e.g. Simon \& Hawley 2009), in the above equation we adopt normalization of variables in terms of the disk scaleheight $H$ and the orbital frequency $\Omega$. Correspondingly, the unit of the magnetic field is $H \Omega \sqrt{4 \pi \rho_{0}}$, and the equilibrium azimuthal field is taken to be $B_{0 y}=0.1$. In this units, our computation domain has 

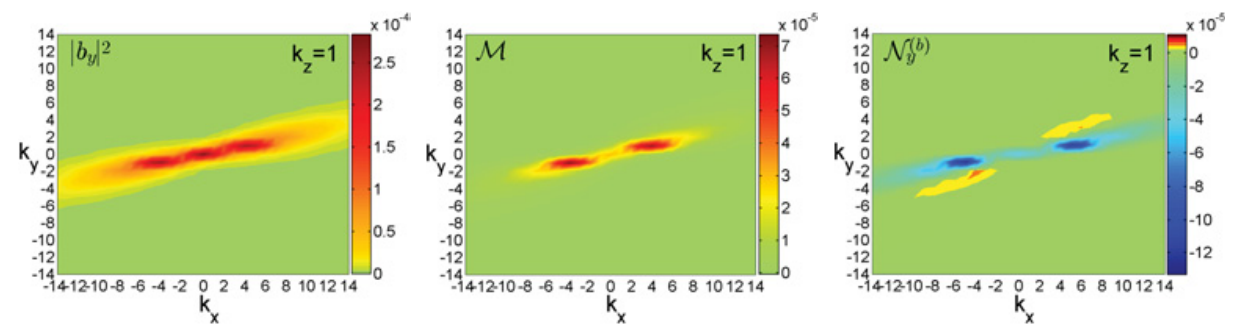

Fig. 6. Spectra of $\left|b_{y}\right|^{2}$, the energy-injecting Maxwell stress, $\mathcal{M}$, and the nonlinear transfer term, $\mathcal{N}_{y}^{(b)}$ at $k_{z}=1$.
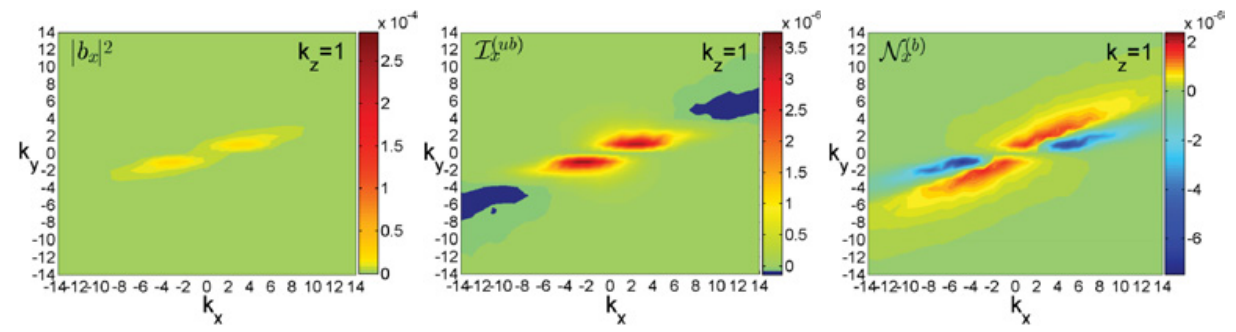

Fig. 7. Spectra of $\left|b_{x}\right|^{2}$, kinetic-magnetic exchange term $\mathcal{I}_{x}^{(u b)}$ and the nonlinear transfer term, $\mathcal{N}_{x}^{(b)}$ at $k_{z}=1$.

size $\left(L_{x}, L_{y}, L_{z}\right)=(4,4,1)$ and the wavenumbers are normalized as $k_{x} L_{x} / 2 \pi \rightarrow k_{x}$, $k_{y} L_{y} / 2 \pi \rightarrow k_{y}$ and $k_{z} L_{z} / 2 \pi \rightarrow k_{z}$. Spectra of kinetic and magnetic energies as well as stresses, integrated in $\left(k_{x}, k_{y}\right)$-plane, show that these quantities achieve largest values for harmonics having small vertical wavenumbers, $k_{z}=0,1,2$. So, here we present spectra of different physical quantities in $\left(k_{x}, k_{y}\right)$-plane just for $k_{z}=1$ : spectra of $\left|b_{y}\right|^{2}$ together with its main sources - the Maxwell stress, $\mathcal{M}$, and $\mathcal{N}_{y}^{(b)}$ - in Figure 6 and spectra of $\left|b_{x}\right|^{2}$ with its sources, $\mathcal{I}_{x}^{(u b)}$ and $\mathcal{N}_{x}^{(b)}$ - in Figure 7. The turbulence is quasi-stationary and all quantities presented in these figures are averaged in time. From these figures it is seen that all - linear and nonlinear - processes are anisotropic. An interplay of these processes with the drift terms ensures the sustenance of the turbulence. The nonlinear terms, $\mathcal{N}_{i}^{(b)}$, transfer $\left|b_{i}\right|^{2}$ from blue areas (where $\mathcal{N}_{i}^{(b)}<0$ ) to yellow and red ones (where $\mathcal{N}_{i}^{(b)}>0$ ) (right plots of Figs. 6 and 7). This fact clearly demonstrates the transverse (and not direct/inverse) nature of the nonlinear cascade processes in the energetically active area in $\mathbf{k}$-space. This central, small wavenumber part of $\mathbf{k}$-space, is the vital area of the turbulence.

From the above-studied three cases, it is obvious that the nonlinear transverse cascade is ubiquitous in shear flows and is a key factor in the sustenance of subcritical turbulence. 


\section{References}

Baggett, J., Driscoll, T., \& Trefethen, L., 1995, Phys. Fluids, 7, 833

Butler, K., \& Farrell, B., 1992, Phys. Fluids, 4, 1637

Chagelishvili, G., Zahn, J.-P., Tevzadze, A., \& Lominadze, J., 2003, A\&A, 402, 401

Chapman J., 2002, J. Fluid Mech., 451, 35

Farrell, B., \& Ioannou, P., 1993, Phys. Fluids A, 5, 1390

Farrell, B., 1988, Phys. Fluids, 31, 2093

Grossmann, S., 2000, Rev. Mod. Phys., 72, 603

Gustavsson, L., 1991, J. Fluid Mech., 224, 241

Horton, W., Kim, J.-H., Chagelishvili, G., Bowman, J., \& Lominadze, J., 2010, Phys. Rev. E, 81, 066304

Jiménez, J., 2013, Phys. Fluids, 25, 110814

Mamatsashvili, G., Gogichaishvili, D., Chagelishvili, G., \& Horton, W., 2014, Phys. Rev. E, 89, 043101

Mamatsashvili, G., Khujadze, G., Chagelishvili, G., Dong, S., Jiménez, J., \& Foysi, H., 2016, Phys. Rev. E., 94, 023111

Reddy, S., Henningson, D., 1993, J. Fluid Mech., 252, 209

Schmid, P., \& Henningson, D., 2000, Stability and Transition in Shear Flows (Springer)

Schmid, P., 2007, Annu. Rev. Fluid Mech., 39, 129

Simon, J., \& Hawley, J., 2009, ApJ, 707, 833

Tevzadze, A., Chagelishvili, G., Zahn, J.-P., 2008, A\&A, 478, 9

Tevzadze, A., Chagelishvili, G., Zahn, J.-P., Chanishvili, R., \& Lominadze, J., 2003, A\&A, 407, 779

Trefethen, L., Trefethen, A., Reddy, S., \& Driscoll, T., 1993, Science, 261, 578

Trefethen, L., \& Embree, M., 2005, Spectra and Pseudospectra: The Behavior of Nonnormal Matrices and Operators (Princeton University Press, Princeton, NJ)

Trefethen, L., 1991, Pseudospectra of matrices, Numerical Analysis, 91, 234

Waleffe, F., 1995, Phys. Fluids, 7, 3060 